41. Patsaev, V. I., Ohanesyan, J., Gurzadyan, G. A. $\quad$ 1972, Soobshcheniya Byrakan. Observ., 45, 20 (in Russian).

Gurzadyan, G. A., Ohanesyan, J. B. $\quad$ 1972, Astron. Astroph., 20, 321; 1972, Sp. Sci. Rev., 13, 647.

42. Cruvellier, P. TR LAS, 69PJ03.

43. Programme FAUST, TR LAS, 70 PJ02.

44. Carruthers, G. R. 1972, Sky Telesc., 44, 218.

45. Navach, C. 1972, Astron. Astroph.,, in press.

46. Kuiper, G. P. private communication.

47. Artsimovitsh, L. A. 1972 , Priroda, No. 9, 2 (in Russian).

APPENDIX I

REPORT FROM LABORATOIRE DE PHYSIQUE STELLAIRE ET PLANÉTAIRE, VERRIÈRES LA BUISSON

R. M. Bonnet

L.P.S.P. is a Laboratory deeply involved in space research. The bulk of its program is Solar Physics and Astronomy in the UV and IR ranges. The period extending from 1970 to 1972 has been characterized by a very intense activity in Balloon, Rocket and Satellite experiments.

\title{
1. The balloon program
}

This program is headed by Dr. P. Lemaire and D. Samain. It takes advantage of the relatively good transparency of the atmosphere in the near UV down to $2750 \AA$ and around $2100 \AA$ at altitudes of $38 \mathrm{~km}$ which are easily reached by the French balloon.

P. Lemaire and D. Samain have designed very high spectral resolution spectrometeres $(20 \mathrm{~m} \AA)$ for the study of the $\mathrm{Mg}$ II resonance lines at $2800 \AA$ and the $\mathrm{C}_{\text {I }}$ forbidden line at $1994 \AA$.

These instruments are made of all-reflective optics and make use of concave gratings as dispersive elements. The use of the double Wadsworth mounting makes it possible to obtain stigmatic spectra of the sun. The instruments are pointed to the sun by means of a biaxial pointing system specially developed at L.P.S.P. In P. Lemaire's instrument, the pointing accuracy is strongly improved up to one arc second by servo controlling the secondary mirror of the Cassegrainian telesope.

Both instruments were launched several times from Aire-sur-L'Adour in France and gave very important and good results (06.071.053).

\section{The rocket program}

L.P.S.P. is also deeply involved in the preparation of rocket experiments. The experiments will be launched before the end of 1972. All experiments deal with the UV spectrum of the sun.

The first experiment will attempt to make photographic pictures of the solar spectrum between Lyman alpha $1216 \AA$ and $2100 \AA$. The instrument is stigmatic and allows a point by point study of the disc surface as well as center to limb studies. The inclusion of a small servo system in the instrument improves the pointing accuracy up to something better than one arc second. The spectral resolution is only $0.3 \AA$.

The purpose of the experiment is to make limb darkening measurements and study the variation in altitude of the inhomogeneities of the solar atmosphere.

The launch is scheduled for October 1972 on a Veronique 61 rocket from French Guyana.

The second experiment will calibrate the solar spectrum with an aimed absolute accuracy of $5 \%$ between $2700 \AA$ and $2900 \AA$. This region includes the two resonance lines of $\mathrm{Mg}$ II and is also very rich in faint absorption lines. Apart from the calibration of Lemaire's balloon spectra of the $\mathrm{Mg}$ II 
lines, it is expected that important information on the unknown source of continuous opacity in the near UV will be obtained.

The instrument is a Czerny-Turner spectrometer which allows a spectral resolution of $16 \mathrm{~m} \AA$. The spatial resolution will be only one to two arc minutes. The calibration has been made on the Black Body model of Dr. Peyturaux at the Institut d'Astrophysique de Paris. There is also provision in the instrument for an inflight calibration on a small mercury source which has been very carefully stabilized within $2 \%$. The launch is scheduled for November 1972 on a Dauphin Rocket from French Guyana.

The third experiment is a very new and original experiment which will share the nose cone of the Dauphin rocket with the previous one.

Its purpose is to measure the profile of the He I line at $584 \AA$.

The instrument is original in that it makes use of a He $\mathrm{r}$ absorption cell in which the pressure is increased linearly. The output signal which is measured by a sodium salicylate window phototube is the result of a convolution between the solar line and the instrumental profile which vary with wavelength. A deconvolution technique is used to reconstruct the shape of the solar line. The spectral resolution is certainly the best one can achieve in this range since it is only limited by the width of the He I line in the cell at room temperature.

\section{The satellite program}

L.P.S.P. has two major satellite experiments in progress: The D2B experiment will be launched on the French D2B satellite in 1975.

Its purpose is to obtain pictures of the sun in preselected wavelengths of the far UV spectrum between $180 \AA$ and Lyman alpha. Due to the limited pointing capability of the spacecraft, the resolution on the disc will be only a few arc minutes. One of the main objectives will be the determination of the chemical composition of the upper atmosphere by means of extinction measurements on the active regions of the sun which, at these wavelengths, can be considered as small individual light source.

The experiment is all made of grazing incidence optics and light is filtered by a combination of grating and metallic filters. The detectors are channeltron photomultipliers.

The OSO-I experiment is the major program of L.P.S.P. The experiment is placed in the pointed section of OSO-EYE which is a considerably improved of the Orbiting Solar Observatories. The instrument is a multichannel high spectral resolution polychromator. It is made of a diffraction limited Cassegrainian telescope and a Czerny-Turner spectrometer.

The telescope is of a special type since the secondary mirror can be actuated in two axes by two small electromagnets in a closed loop system and generates spatial scan of the sun. It is possible to obtain images of one arc minute sides with a spatial resolution of one arc second on the sun. This imaging possibility supplements those of the spacecraft itself.

The spectrometer is a six channel polychromator centered on the $\mathrm{H}$ and $\mathrm{K}$ lines of $\mathrm{Ca} \mathrm{Il}$, the two resonance lines of $\mathrm{Mg}$ II, Lyman alpha and Lyman Beta. The spectral resolution is adjustable and can reach $20 \mathrm{~m} \AA$ at best and $1 \AA$ at the worst. The spectrum is scanned by rotation of the plane grating when the instrument is pointing at any point on the sun. The experiment will be operated in near real time on a one orbit basis, thus providing the capability of modifying the observing program and adapting it to the extension of structures on the disc.

The major purpose of the experiment is to investigate the solar chromosphere with the highest spatial (one arc second), spectral $(20 \mathrm{~m} \AA)$ and temporal $(160 \mathrm{~ms})$ resolutions both in the quiet and active regions. The observation of all six lines simultaneously will allow detailed model investigations for areas of one arc second on the sun. Temporal and transient phenomena such as the 5 minutes oscillations and flares will be also throroughly investigated.

The experiment has also the capability of looking at stars during the night portions of the orbit.

The instrument will be operated in a way similar to ground-based observatories. A guest investigator program has been planned by the experimenters to manage data reduction and interpre- 
tation. Guest investigators are selected on the basis of a proposed observing program. It is expected that guest investigators will be involved in the operation of the experiment in real time from the experiment control center which will be located at Boulder (Colo. USA).

The launch of the experiment is scheduled for May 1973.

\section{Laboratory investigations related to astrophysics}

Under N. Coron, L.P.S.P. is also developing very high sensibility far IR detectors of the cooled bolometer type. A star detectivity of $5 \times 10^{12} \mathrm{~W}^{-1} \mathrm{~cm} \times V \mathrm{~Hz}$ is currently achieved. Observing programs of astrophysical interest are in progress in cooperation with Dr. P. Lena at Meudon observatory. Let us quote in particular, the observation of the Galactic Center from a plane in the submillimetric range.

\section{APPENDIX II}

\section{DEVELOPMENT OF NEW INSTRUMENTS AND TECHNIQUES IN THE SOLAR SATELLITE PROJECT AT HARVARD COLLEGE OBSERVATORY}

\section{A. Dalgarno}

The solar satellite project is currently engaged on the development of the following flight instruments:

1. A sounding rocket spectrometer to study the emission from the quiet sun in the wavelength range $1550 \AA$ to $1660 \AA$ with a resolution of $0.03 \AA$. This instrument was flown successfully on July 27, 1972, and a further flight after modification is planned for February 1973 to study the wavelength range from $1850 \AA$ to $2300 \AA$ with a resolution of $0.06 \AA$.

2. An extreme ultraviolet (EUV) spectroheliometer to be flown on the Apollo Telescope Mount (ATM) of the Skylab space station which is scheduled for launch in May 1973. This instrument is designed to obtain up to seven simultaneous spectroheliograms in the range $280 \AA$ to $1340 \AA$ with a spatial resolution of $5 \mathrm{arc} \mathrm{sec}$, , as well as spectra with a resolution of $1.6 \AA$ over the same wavelength range.

3. Three sounding rocket spectroheliometers to be flown as part of the calibration program for the Skylab spectroheliometer. These instruments will cover the same wavelength range and have the same spectral resolution as the Skylab instrument and will be used to re-establish its photometric calibration by simultaneous measurement of the emission from a quiet area near the center of the solar disc. The three calibration flights are currently planned for the manned periods of Skylab operation during May, August and November 1973.

The laboratory program supporting these photoelectric flight instruments has been aimed at improving the quality of the standards available for photometric calibration over the wavelength range $300 \AA$ to $2500 \AA$ and also at developing detectors of increased sensitibity and stability over the same wavelength range. The aim of the laboratory calibration program is to establish an absolute photometric accuracy for the rocket instruments of better than $\pm 10 \%$ during flight and to attempt to maintain a similar accuracy for the satellite instrument during the orbital lifetime. For this purpose, a series of laboratory standards are being developed having pedigrees directly traceable to the National Bureau of Standards. The standard light sources used in the laboratory are Eppley total irradiance tungsten filament lamps emitting in the visible and 'Penray' mercury lamps used in conjunction with interference filters at $2537 \AA$. A Samson type, rare gas, double ionization chamber is used as the absolute standard detector at EUV wavelengths and a Reeder gold-black thermopile is used as a primary standard detector to transfer a calibration from the visible standard sources to the EUV detectors. Transfer standards in use are open tungsten diodes for the wavelength range $300 \AA$ to $1400 \AA$ and a sealed Spicer Cs-Te diode with a $\mathrm{MgF}_{2}$ window for the wavelength range 